\title{
Comparison of the Effects of Bilateral Orbital Prefrontal Cortex Lesions and Amygdala Lesions on Emotional Responses in Rhesus Monkeys
}

\author{
Alicia Izquierdo, Robin K. Suda, and Elisabeth A. Murray \\ Section on the Neurobiology of Learning and Memory, Laboratory of Neuropsychology, National Institute of Mental Health, National Institutes of Health, \\ Bethesda, Maryland 20892
}

\begin{abstract}
The present study examines the effects of bilateral orbital prefrontal cortex (PFo) lesions on monkeys' emotional responses in two different contexts: in the presence of a rubber snake and in the presence of a human intruder. For comparison, we also assessed the responses of rhesus monkeys with selective amygdala lesions on these same tasks. Monkeys with PFo lesions, like those with amygdala lesions, displayed blunted emotional responses to the fake snake. Unlike monkeys with amygdala lesions, however, monkeys with PFo lesions displayed more mild aggression than controls in the presence of a human intruder. The findings support the idea that the PFo helps integrate sensory signals in the service of choosing among competing responses. In addition, they point to a divergence of the roles of the PFo and amygdala in responding to a social stimulus, the human intruder.
\end{abstract}

Key words: fear; response selection; decision making; aggression; inhibitory control; phobia

\section{Introduction}

Studies of orbital prefrontal cortex (PFo) function have emphasized its role in rule-based response selection, inhibitory control, and representation of the affective value of goals (Holland and Gallagher, 2004). In addition, the PFo is thought to play a role in social and emotional behavior. Damage to the PFo and amygdala produce similar effects in many instances, for example, on tests of reinforcer devaluation in monkeys (Malkova et al., 1997; Izquierdo et al., 2004) and rats (Hatfield et al., 1996; Gallagher et al., 1999) and on the Iowa gambling task in humans (Bechara et al., 1999). In some cases, adaptive response selection requires the PFo and amygdala to functionally interact (Baxter et al., 2000). These findings inform the roles of the PFo and amygdala in appetitive tasks but leave unaddressed their potential interaction in emotional behavior.

In macaques, there is little information available on the role of the PFo in responding to negative events predicted by environmental cues, including potential predators and social threats. One tool that might be used to investigate this issue relies on monkeys' innate emotional responses to snakes. Macaques, even

\footnotetext{
Received March 30, 2005; revised July 15, 2005; accepted July 29, 2005.

This work was supported by the Intramural Research Program of the National Institute of Mental Health. We thank R. Saunders and the staff of the Nuclear Magnetic Resonance Facility, National Institute of Neurological Disorders and Stroke, for assistance obtaining MR scans, P.-Y. Chen for histological support, and E. Buch and G. Edler for help with behavioral testing.

Correspondence should be addressed to Dr. Alicia Izquierdo, Laboratory for Integrative Neuroscience, National Institute on Alcohol Abuse and Alcoholism, National Institutes of Health, 5625 Fishers Lane, Room 2N09, Rockville, MD 20852. E-mail: izquiera@mail.nih.gov.

DOI:10.1523/JNEUROSCI.1232-05.2005

Copyright $\odot 2005$ Society for Neuroscience $\quad$ 0270-6474/05/258534-09\$15.00/0
}

snake-naive ones, exhibit marked defensive responses in the presence of fake or real snakes (Mineka, 1987; Nelson et al., 2003), and elicitation of these responses requires an intact amygdala (Aggleton and Passingham, 1981; Meunier et al., 1999, Kalin et al., 2001). Another commonly used tool relies on monkeys responses to an unfamiliar human observer. Early investigations by Butter et al. $(1968,1970)$ found evidence of increased defensive behaviors in monkeys with PFo lesions in response to a human observer and a doll but not in response to a fake snake. In contrast, Kalin et al. (2001) found that monkeys with bilateral amygdala lesions displayed fewer defensive behaviors in the presence of a snake but not in response to a human observer. This set of findings is seemingly at odds with the evidence for functional interactions of the amygdala and the PFo in other settings (Baxter et al., 2000; Schoenbaum et al., 2003). To further complicate matters, yet other studies (Butter et al., 1970; Butter and Snyder, 1972 ) report an overall reduction in aggressive behaviors in monkeys with PFo lesions in the presence of a human observer and somewhat less of a reduction in aggression in response to a doll or snake.

Given the foregoing, we attempted a more systematic investigation of the effects of PFo damage on emotional behavior. Rhesus monkeys with bilateral PFo lesions were assessed for their emotional responses in two different contexts: in the presence of a fake snake and in the presence of a human intruder. For comparison, we evaluated monkeys with bilateral excitotoxic amygdala lesions on the same tasks. These studies were intended to inform the role of the PFo and the amygdala, and their potential interaction, in response selection. If the PFo and the amygdala worked together to support responses in the presence of emotionally charged stimuli, we would predict similar effects of PFo 
and amygdala lesions. If not, results from the two experimental groups might diverge.

\section{Materials and Methods \\ Experiment 1: responses to fake snake, spider, and neutral objects}

Snakes produce significant behavioral reactions in both snake-naive and snake-experienced rhesus monkeys (Mineka, 1987; Nelson et al., 2003). Thus, exposure to fake or real snakes provides robust measures of emotional responses without the need for formal training. Although several studies have measured emotional responses to a snake, our study differs from most of these in that withdrawal responses from the snake were pitted directly against approach responses to familiar foods. In addition, testing was performed only after monkeys had participated in other cognitive tasks in the same apparatus. Thus, all monkeys had extensive experience displacing objects to obtain food rewards. Because the monkeys' prepotent response was to approach the test tray to obtain food rewards, our method arguably provided a more sensitive measure of response selection relative to other tests in which monkeys have been exposed to fake or real snakes. We collected two measures. First, we recorded latencies to retrieve a food reward located on top of a clear Plexiglas box containing either a neutral "junk" object or a potentially feared object. Second, monkeys' facial expressions and body movements in response to such stimuli were videotaped and analyzed.

\section{Subjects}

Eighteen rhesus monkeys (Macaca mulatta), all male, were used. Four monkeys had bilateral aspiration lesions of the orbital prefrontal cortex (group PFo), four monkeys had bilateral excitotoxic lesions of the amygdala (group Amyg), and 10 were maintained as unoperated controls (group Con). Of the 10 unoperated monkeys, four were tested concurrently with group Amyg and six with group PFo. Monkeys with bilateral PFo lesions and their controls were the same as those studied by Izquierdo et al. (2004). Before the present experiments, the monkeys had been given several cognitive tests not reported here. The training histories of the monkeys in the different groups (group PFo and their controls vs group Amyg and their controls) were highly similar, having included food preference tests, visual discrimination learning, and tests of reinforcer devaluation. The monkeys weighed $6.2-12.6 \mathrm{~kg}$ at the beginning of the study, were housed individually in rooms with automatically regulated lighting (12 h light/dark cycle; lights on at 7:00 A.M.), and were maintained on primate chow (catalog \#5038; PMI Feeds, St. Louis, MO) supplemented with fresh fruit. Monkeys were maintained on a controlled diet to ensure sufficient motivation and healthy body weight. Water was available ad libitum.

\section{Apparatus}

Monkeys were trained in a modified Wisconsin general testing apparatus (WGTA) located in a darkened room. A clear Plexiglas box measuring $11.4 \mathrm{~cm}$ (width) $\times 71.1 \mathrm{~cm}$ (length) $\times 11.4 \mathrm{~cm}$ (height) was placed within the test compartment of the WGTA. The box was hinged at the back, which allowed the experimenter to easily lift the top and place objects within the box. Test objects included the following: a rubber snake measuring $50.8 \mathrm{~cm}$ in length and $\sim 2 \mathrm{~cm}$ in diameter, a rubber "jumping" spider measuring $10 \mathrm{~cm}$ (width) $\times 13.5 \mathrm{~cm}$ (length) $\times 2.5 \mathrm{~cm}$ (height), made to jump by an air bladder, or one of eight neutral objects, which, like the snake and jumping spider, were novel at the beginning of the experiment. Three additional novel objects were dedicated to the accommodation phase (see below). Food rewards consisted of a "fruit snack" (Giant Food, Landover, MD), a chocolate candy (M\&Ms; Mars Candies, Hackettstown, NJ), or one-half of a peanut.

Test sessions were videotaped from two vantage points. One camera was located on top of the WGTA facing straight down, thereby providing a view of the test compartment from above. A second camera was located behind the tester ( $\sim 1 \mathrm{~m}$ from the monkey cage) to record a frontal view of the monkey.

\section{Surgery}

At the time of surgery, anesthesia was induced with ketamine hydrochloride $(10 \mathrm{mg} / \mathrm{kg}$, i.m.) and maintained with isoflurane (1.0-3.0\%, to ef- fect). The animals received $0.45 \%$ sodium chloride plus $5 \%$ dextrose via an intravenous drip. Aseptic procedures were used. Heart rate, respiration rate, blood pressure, expired $\mathrm{CO}_{2}$, and body temperature were monitored throughout the procedure. After the aspiration removal (orbital prefrontal cortex) or injections of excitotoxin (amygdala) were completed, the wound was closed in anatomical layers.

All monkeys received a preoperative and postoperative treatment regimen consisting of dexamethasone sodium phosphate $(0.4 \mathrm{mg} / \mathrm{kg})$ and Cefazolin antibiotic $(20 \mathrm{mg} / \mathrm{kg}$; Watson Laboratories, Corona, CA) for $1 \mathrm{~d}$ before surgery and 1 week after surgery to reduce swelling and prevent infection, respectively. At the end of surgery, and for 2 additional days, the monkeys received the analgesic ketoprofen (10-15 mg). Ibuprofen $(100 \mathrm{mg}$ ) was provided for 5 additional days.

Surgeries were performed in two stages. We used the same method described in previous reports from this laboratory (Malkova et al., 1997; Baxter et al., 2000; Izquierdo and Murray, 2004). Two monkeys (PFo-1 and $\mathrm{PFo}-3$ ) received removal of the left orbital prefrontal cortex as the first operation, whereas the two others (PFo-2 and $\mathrm{PFo}-4)$ received removal on the right side first. Monkeys with excitotoxic amygdala lesions also received counterbalanced, two-stage surgeries: Amyg-2 and Amyg-4 received injections in the right hemisphere as the first operation, and Amyg-1 and Amyg-3 received injections in the left hemisphere first. The second stage surgery took place an average of 3.3 months after the first stage surgery.

Amygdala lesion by ibotenic acid injection. After induction of anesthesia, monkeys were placed in a stereotaxic frame. A bone flap extending over the midline was made in the appropriate portion of the cranium, and a final reading was taken on the position of the sagittal sinus, which served as the landmark for calculation of stereotaxic coordinates in the mediolateral dimension. Slits were cut in the dura to allow passage of the injection needle. Injections of ibotenic acid were placed stereotaxically throughout the amygdala in one hemisphere, with coordinates determined from magnetic resonance imaging (MRI) scans performed an average of $2.75 \mathrm{~d}$ before the first surgery and $1.5 \mathrm{~d}$ before the second surgery. Fifteen to 19 injections, each consisting of $0.6-1.0 \mu \mathrm{l}$ of ibotenic acid $(10 \mathrm{mg} / \mathrm{ml}$; Biosearch Technologies, Novato, CA), were made into the amygdala via the 30 gauge needle of a Hamilton syringe held in a micromanipulator. The injection sites were $\sim 2 \mathrm{~mm}$ apart in each plane. Each injection was made at the rate of $0.2 \mu \mathrm{l} / \mathrm{min}$, and the needle was left in place 2-3 min after each injection to limit diffusion of the toxin up the needle track. The intended lesion (Fig. 1) included the entire amygdala, including both the basolateral nuclear group as well as the central, medial, and cortical nuclei.

Orbital prefrontal cortex lesion by aspiration. A half-moon-shaped craniotomy was performed over the region of the prefrontal cortex. The dura mater was cut near the dorsal edge of the bone opening and reflected ventrally. Using a combination of suction and electrocautery, the orbital prefrontal cortex was removed by subpial aspiration through a finegauge metal sucker, insulated except at the tip. The intended lesion (Izquierdo et al., 2004) extended from the fundus of the lateral orbital sulcus, which marked the lateral boundary of the lesion, to the fundus of the rostral sulcus, medially. The rostral limit of the lesion was a line joining the anterior tips of the lateral and medial orbital sulci, and the caudal limit of the lesion was $\sim 5 \mathrm{~mm}$ rostral to the junction of the frontal and temporal lobes. Thus, the lesion included Walker's areas 11, 13, and 14 and the caudal part of area 10 (Walker, 1940).

\section{Assessment of the lesions}

The lesions in all eight operated monkeys were assessed quantitatively from postoperative MRI scans. The extent of amygdala damage was evaluated from T2-weighted scans obtained within $12 \mathrm{~d}$ of surgery, and the extent of orbital prefrontal cortex damage from T1-weighted scans was obtained an average of 11.3 months after surgery.

For each operated animal, MR scan slices were matched to drawings of a standard rhesus monkey brain at $1 \mathrm{~mm}$ intervals. Each lesion was subsequently plotted onto the standard sections. For amygdala lesions, the region of hypersignal evident in the T2-weighted MR scan was plotted onto the standard sections. The extent of hypersignal has been reported to accurately reflect the extent of neuronal cell loss, at least in the 
hippocampus (Malkova et al., 2001; Nemanic et al., 2002). For the orbital prefrontal cortex lesions, the extent of the lesion visible in the T1-weighted scan was plotted. We then measured the volume of the lesion as a function of the total volume of the structure (either amygdala or orbital prefrontal cortex) in the standard.

In all four subjects of group PFo, damage to the orbital prefrontal cortex was essentially as intended. The lesions removed an average of $78.7 \%$ of the PFo bilaterally (range, 69.2-88.9). The removals systematically spared a narrow strip of cortex immediately ventral to the rostral sulcus, a region classified as infralimbic cortex by Preuss and Goldman-Rakic (1991). For monkeys in group Amyg, the damage was also essentially as intended. Left amygdala damage could not be assessed for one case (Amyg-2), so the estimates are based on seven of the eight amygdalas in the four subjects. We estimate the lesion to include $91.5 \%$ of the amygdala bilaterally (range, 85.2-100). Each of the monkeys with amygdala lesions sustained some inadvertent damage to adjacent structures. Amyg-1, the monkey with the most complete lesion, sustained bilateral unintended damage to anterior portions of the entorhinal cortex and hippocampus, to portions of the ventral claustrum, the substantia innominata, and piriform cortex. The remaining monkeys in this group sustained only minor and unilateral damage to a subset of these regions. (Note that only one hemisphere could be evaluated in case Amyg-2.) The percentage of damage to the target structures in each hemisphere for each individual monkey is provided in Table 1.

\section{Behavioral testing procedures}

Accommodation. Before the main task, all monkeys were accommodated to the camera, Plexiglas box, and general test setup in the WGTA over two sessions. In the first session, monkeys were required to retrieve a food reward located in a food well of the test tray while the screen that normally separated the monkey from the experimenter stayed open for videotaping. During the second session, monkeys were exposed to the Plexiglas box; all monkeys readily reached for the food reward 20 times while the box was empty and 10 times with one of the three novel objects dedicated to this phase inside.

Main task. The method was adapted from Mineka and her colleagues (Mineka et al., 1980; Mineka, 1987) and is identical to that reported previously (Izquierdo and Murray, 2004). On each trial, the monkeys were allowed to reach for and to procure a food reward that had been placed on top of the clear box. The food was always located at the center of the back edge of the top, the edge nearest the experimenter, which meant that the monkey had to reach over the object in the Plexiglas box to obtain the food reward. To help the experimenter quickly and accurately set out the food, a small " $x$ " marked the spot.

Each session was comprised of 10 trials. Eight of the trials were those in which an originally novel, neutral object was placed in the clear box. Each object was used once per session; thus, a different object appeared on each neutral object trial within a session. For the remaining two trials, the rubber snake and the rubber jumping spider were used. The snake and spider trials appeared pseudorandomly in the sequence of 10 trials, with the constraint that neither appeared on the first trial of the session. For each monkey, a single food (one of the foods that ranked highly during a previous test of food preference) was assigned to be used throughout the experiment.

During the intertrial interval, while the screen between the monkey compartment and test compartment blocked the monkey's view of the
Table 1. Percentage of damage to orbital prefrontal cortex (group PFo) and amygdala (group Amyg)

\begin{tabular}{lccc}
\hline Monkey & Left & Right & Mean \\
\hline PFo-1 & 92.5 & 85.2 & 88.9 \\
PFo-2 & 76.1 & 62.2 & 69.2 \\
PFo-3 & 85.4 & 72.9 & 79.2 \\
PFo-4 & 85.0 & 70.0 & 77.5 \\
Amyg-1 & 100 & 100 & 100 \\
Amyg-2 & & 98.5 & 98.5 \\
Amyg-3 & 94.7 & 77.3 & 86.0 \\
Amyg-4 & 100 & 70.4 & 85.2 \\
\hline
\end{tabular}

Numerals indicate percentage of damage to orbital prefrontal cortex and amygdala sustained by each of the operated monkeys. PFo-1 to PFo-4, Monkeys with bilateral removals of the orbital prefrontal cortex by aspiration; Amyg-1 to Amyg-4, monkeys with excitotoxic amygdala lesions; Left, left hemisphere; Right, right hemisphere; Mean, average of the values for the left and right hemispheres.

Plexiglas box, the experimenter loaded the box and set out the food. A trial was initiated when the experimenter raised the opaque screen. During this part of the trial, the experimenter faced the video monitors (located to one side of the WGTA), and only her profile was visible to the monkey. If the monkey took the food, the screen was immediately lowered, terminating the trial. If the monkey failed to take the food within $30 \mathrm{~s}$, the trial was terminated. Monkeys were tested for a total of five sessions, each consisting of 10 trials separated by 20 s intervals, at a rate of one session every other day.

Videotape analysis. Time code generators on the videotape recorders were used to synchronize the two camera views. In this way, the monkeys' latencies to reach for the food reward (derived from the top-down view), and the duration and frequency of their behavioral reactions to the stimuli (derived from the frontal view) could be analyzed to the nearest millisecond. 
Table 2. Behaviors analyzed during experiment 1 (snake task) and experiment 2 (human intruder task)

\begin{tabular}{ll}
\hline Behavior & Description \\
\hline Mild aggression & \\
Frown & Wrinkles or moves eyebrows up and down \\
Ears back & Flattens ears against head \\
Yawn & Opens mouth wide, baring upper teeth \\
High aggression & \\
Head/body lunge & Thrusts head or body forward \\
Cage shake & Shakes cage \\
Mouth threat & Opens mouth slightly, exposing lower teeth \\
Defense & \\
Freezing & Motionless for $\geq 3$ s \\
Startle & Jerks suddenly \\
Eye/head aversion & Avoids eye contact, shifts gaze or whole head \\
Piloerection & Hair stands on end \\
Move away & Retreats from the stimulus \\
Submission & \\
Lip smack & Purses and alternatively closes and opens lips \\
Grimace & Mouth closed, pulls lips backward exposing teeth \\
Presentation & Presents its hindquarters with tail up \\
Approach & \\
Look at & Makes eye contact \\
Move toward & Shifts body forward, closer to stimulus \\
$>$ Touch & Handles with hand or foot \\
Take/eat reward & Picks up or mouths the food reward \\
Other behaviors (not directed toward the stimulus) & \\
Manual exploration & Handles any part of its surrounding \\
Oral exploration & Licks or mouths any part of its surrounding \\
Locomotor stereotypies & Activities, such as circling, hopping, repeated three or more times \\
Self-directed activities & Scratches, grooms, holds, etc. any part of its body \\
Look away & Looks away while engaged in behavior not directed toward stimulus \\
Teeth gnashing & Chewing motion without food in mouth \\
Miscellaneous & Engages in any peculiar activity not described above \\
\hline &
\end{tabular}

Videotaped food-retrieval latencies and emotional behavior were scored independently by two viewers. Timing for the latency measure was initiated when the WGTA screen was raised above a given point, located $\sim 15 \mathrm{~cm}$ above the test tray, which was marked on the front of the cage and visible in the frontal camera view. The response was considered completed when the monkey grasped the food reward, just before it withdrew its arm. If no response was made within the $30 \mathrm{~s} \mathrm{time} \mathrm{limit,} \mathrm{a}$ score of $30 \mathrm{~s}$ was given.

With minor modification (the inclusion of teeth gnashing as an other behavior), behavioral scoring methods were the same as those used by Meunier et al. (1999). A list of the behavioral categories, together with a brief description of the constituent behaviors, is provided in Table 2.

For each monkey, behaviors during each of the five snake trials were analyzed for mean cumulative duration and mean cumulative frequency (Meunier et al. 1999; Izquierdo and Murray, 2004). Subsequently, means for each group were obtained. Behaviors observed during the snake trials were grouped as either defensive (e.g., move away, freezing, and eye/head aversion) or approach (move toward and take reward). Because the "look at" behavior was observed in monkeys that never reached over the rubber snake as well as in monkeys that reached quickly, this behavior was excluded as an approach behavior. In addition, because the duration of the snake trial varied across monkeys, for purposes of analysis, each trial was prorated to a 30 s interval, which was the maximum trial length observed. The frequencies were kept as raw scores.

Videotapes were first scored by an observer who was aware of the group assignments. A subset of sessions was subsequently scored independently by a second observer, one who was unaware of group assignments. Interobserver reliability was calculated using Pearson's correlation coefficients and averaged across the five sessions per monkey in a sample of six monkeys (two monkeys from each of the operated groups and four monkeys from the control group). In general, there was good agreement between the two scorers (analysis of both duration and frequency of defensive and approach behaviors in a subset of group Amyg and group Con, all correlations $>0.939$, all $p<0.022$; in a subset of group PFo and group Con, all correlations $>0.957$, all $p<0.043)$. This suggests that behaviors exhibited by the monkeys in response to the rubber snake could be reliably identified and distinguished from one another. More importantly, however, high reliability between a scorer blind to the group assignments and one aware of the group assignments strongly argues against the possibility that any group differences observed are caused by experimenter bias.

\section{Experiment 2: responses to human intruder}

The response of intact rhesus monkeys to the stare of an unfamiliar human intruder produces emotional behavior characterized by defensive, submissive, and aggressive behaviors (Kalin and Shelton, 1989). Although Kalin et al. (2001) found no effect of bilateral amygdala lesions on this measure, we included this task in our battery to measure emotional responses to a social stimulus, which would complement our evaluation in the snake task of reactions to a potential predator. In addition, we reasoned that even if the behavior exhibited in response to a human intruder was unaffected by the lesions, it would provide a potential control for the production of emotional responses in the presence of the snake.

\section{Subjects}

These were the same subjects as in experiment 1 , with the exception that, after completing experiment 1 , one monkey was killed because of illness that did not respond to treatment. Thus, in experiment 2, there were three monkeys in group PFo instead of four.

\section{Apparatus}

We used the same videotaping apparatus as in experiment 1 , but only the frontal camera view was used. Also, for the human intruder task, monkeys were not tested in the WGTA but in the open space of an unfamiliar room. One male human, never before seen by the monkeys, served as the human intruder.

\section{Test procedures}

Test procedures and behavioral scoring methods are identical to those reported by Izquierdo and Murray (2004). Monkeys were placed in their usual test cage, taken to a room they had never been in, and left alone for 5 min [alone condition (Alone)]. A human male unfamiliar to the monkey then entered the room, sat $\sim 2.5 \mathrm{~m}$ away from the cage, and presented his profile to the monkey for $5 \mathrm{~min}$. The human never made eye contact [no eye contact condition (NEC)] with the monkey during this time. After leaving the room for $3 \mathrm{~min}$, the same human returned to the room, sat $2.5 \mathrm{~m}$ away from the monkey, and proceeded to stare at the monkey [stare condition (ST)] for $5 \mathrm{~min}$. In the ST condition, the human remained motionless and projected a neutral face toward the monkey. All conditions were videotaped and analyzed for duration of behavior in seconds, using the same scoring methods as in experiment 1 . As was the case for behavioral scoring in experiment 1 , interobserver reliability was high (analysis of a subset of group Amyg and group Con, all correlations $>0.976$, all $p<0.01$; analysis of a subset of group PFo and group Con, all correlations $>0.934$, all $p<0.05$ ). 
Results

Experiment 1

Responses to fake snake, spider, and neutral objects

Because no statistical differences were found between the two control groups in any of our measures, these groups were collapsed into a single group Con for all analyses. Food-retrieval latencies for the three trial types are shown in Figure 2. A three-by-three-by-five ANOVA of the response latencies by group (Con, Amyg, and PFo) with repeated measures on trial type (snake, spider, and neutral object trial types) and session (1-5) revealed a significant interaction between group and trial type $\left(F_{(4,30)}=4.571 ; p=0.005\right)$. Statistical analyses resulting from the ANOVA are summarized in Table 3. Separate follow-up ANOVAs showed that group differences in food-retrieval latency emerged on snake and spider trials (snake, $F_{(2,15)}=9.738, p=0.002$; spider, $\left.F_{(2,15)}=6.350, p=0.010\right)$ but not on neutral object trials $\left(F_{(2,15)}=1.372 ; p=0.284\right)$. In addition, there was a decrease in latency across the five sessions, and this was true for all three trials types $\left(\right.$ snake, $F_{(4,60)}=8.892, p<0.001$; spider, $F_{(4,60)}=20.149$, $p<0.001$; neutral objects, $\left.F_{(4,60)}=3.490, p=0.013\right)$. There were no significant interactions of session and group (snake, $F_{(8,60)}=$ $1.255, p=0.284$; spider, $F_{(8,60)}=0.672, p=0.714$; neutral objects, $\left.F_{(8,60)}=0.637, p=0.744\right)$.

Snake trials. Food-retrieval latencies on snake trials are shown in Figure $2 A$. On average, control monkeys displayed long latencies to retrieve the food on snake trials. On many trials, they never reached for the food, and on others they took a long time to do so. In contrast, the monkeys in both operated groups displayed relatively shorter food-retrieval latencies. Individual ANOVAs conducted on each session revealed that latencies differed significantly by group for all five sessions ( $\operatorname{session~} 1, F_{(2,15)}=4.390, p=$ 0.032 ; session $2, F_{(2,15)}=7.077, p=0.007$; session $3, F_{(2,15)}=$ $10.745, p=0.001$; session $4, F_{(2,15)}=5.613, p=0.015$; session 5 , $\left.F_{(2,15)}=5.878, p=0.013\right)$. Group PFo, although not significantly different from the controls on the first session (Bonferroni's test, $p=1.0$ ), showed a marginal difference from the controls on the second session (Bonferroni's test, $p=0.060$ ) and differed significantly from controls on the last three sessions (Bonferroni's tests, sessions 3-5, respectively: $p=0.007, p=0.052, p=0.044$ ). For all five sessions, the group Amyg showed faster food retrieval relative to the controls (Bonferroni's tests, sessions $1-5$, respectively: $p=0.029, p=0.013, p=0.006, p=0.049, p=0.045$; Amyg vs Con). Post hoc tests revealed no significant differences between group Amyg and group PFo in latencies for all five sessions.

Spider trials. Food-retrieval latencies on spider trials are shown in Figure $2 \mathrm{~B}$. When confronted with the spider, the responses of the groups appeared similar; all monkeys displayed longer latencies in the initial session relative to later sessions. Still, a group difference emerged for session $1\left(F_{(2,15)}=5.314 ; p=\right.$ 0.018 ), and post hoc Bonferroni's tests revealed that group Amyg displayed faster food-retrieval latencies than group Con $(p=$ $0.017)$ but did not differ from group PFo $(p=0.411)$.

\section{Behavior during snake trials}

Defensive behavior. Figure 3 shows the mean cumulative duration of defense and approach behaviors exhibited by each group dur-
B

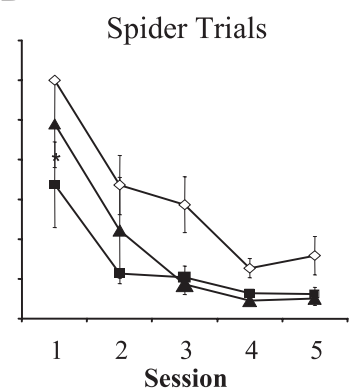

C

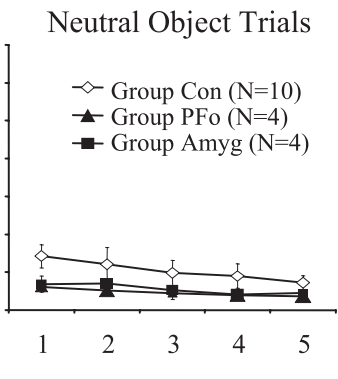

Table 3. Summary of three-by-three-by-five ANOVA on food-retrieval latencies in experiment 1

\begin{tabular}{lrr}
\hline & \multicolumn{1}{c}{} & $p$ value \\
\hline Group & 10.572 & 0.001 \\
Trial type & 13.149 & $<0.001$ \\
Group-by-trial type & 4.571 & 0.005 \\
Session & 26.296 & $<0.001$ \\
Trial type-by-session & 7.031 & $<0.001$ \\
Session-by-group & 1.145 & NS \\
Trial type-by-session-by-group & 0.806 & NS \\
\hline
\end{tabular}

Resulting $F$ and $p$ values of a three (Con, Amyg, PFo)-by-three (snake, spider, neutral object)-by-five (session 1-5) ANOVA on food-retrieval latencies in experiment 1. NS, Not significant.

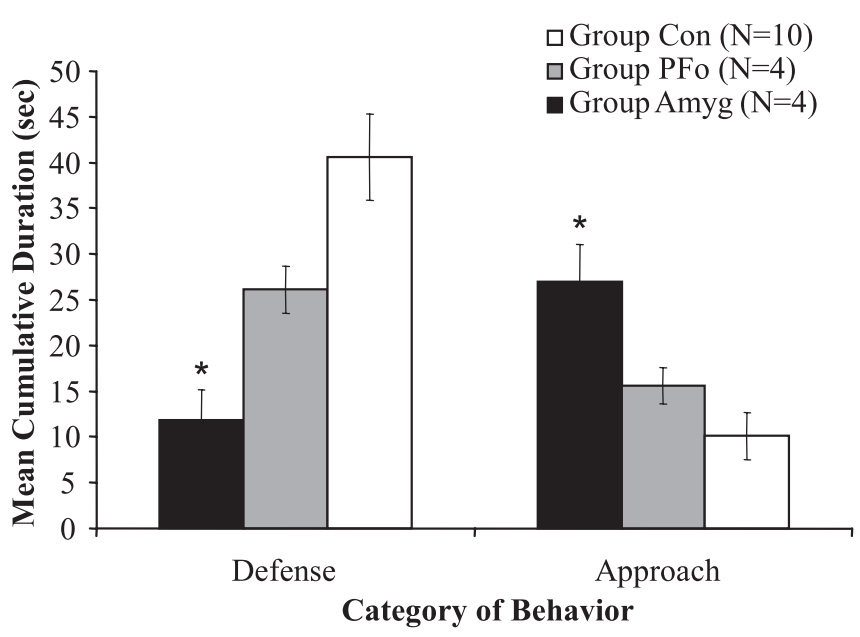

Figure 3. Group mean cumulative duration of defense and approach behaviors when exposed to the rubber snake. Only group Amyg differed significantly from group Con in both their defensive and approach behaviors. * Significantly different from controls ( post hoc Bonferroni's tests, $p<0.05$ ). Group Con, $n=10$; group PFo, $n=4$; group Amyg, $n=$ 4. Error bars indicate SEM.

ing rubber snake exposure. In general, both operated groups displayed fewer defensive behaviors than did the controls. Whereas the monkeys in group Con exhibited defensive behaviors for $41.8 \mathrm{~s}$ per session, on average, monkeys in group Amyg displayed only $11.8 \mathrm{~s}$ and those in group PFo only $26.1 \mathrm{~s}$ of defensive behavior. (Mean cumulative numbers could be greater than the $30 \mathrm{~s}$ trial duration because durations for constituent behaviors in the defensive category were summed.) A repeated-measures ANOVA on defensive behavior across all five sessions resulted in a main effect of group $\left(F_{(2,15)}=9.402 ; p=0.002\right)$ and a within-subjects 

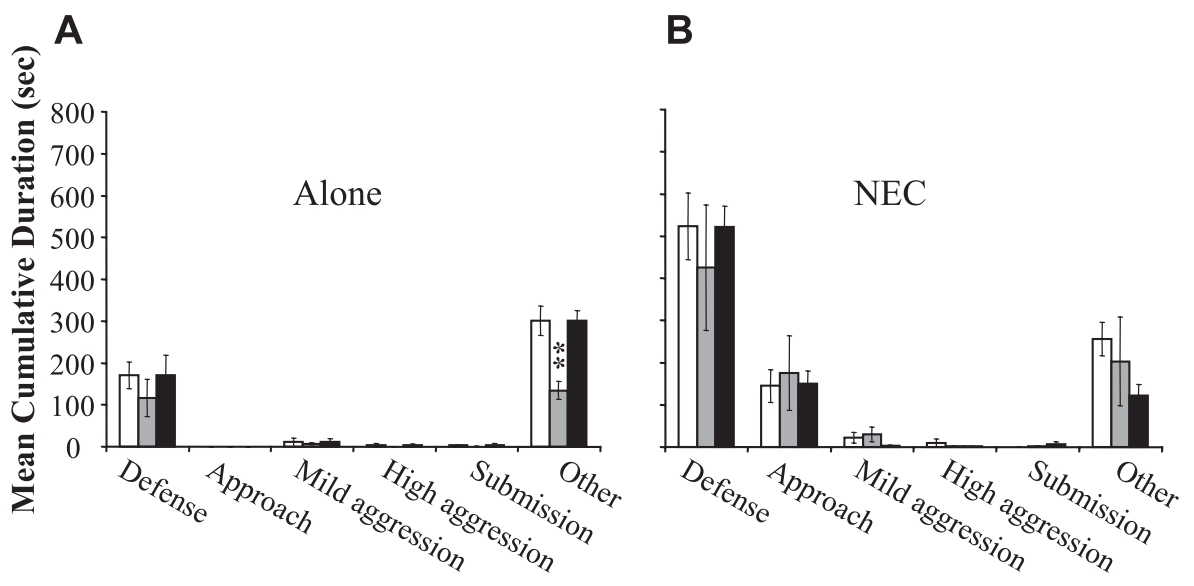
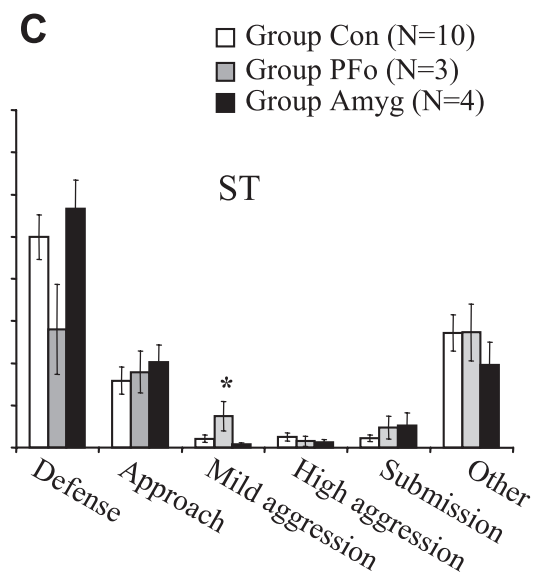

\section{Category of Behavior}

Figure 4. Responses to a human intruder. The mean cumulative duration of categories of behavior during the human intruder task is shown. Error bars indicate SEM. $\boldsymbol{A}$, Alone condition; $\boldsymbol{B}$, NEC condition; C, ST condition. Group PFo showed less other behaviors in the Alone condition and more mild aggression in the ST condition. For a more detailed description of behaviors, see Table 2. *Significantly different from both group Con and group Amyg. ${ }^{*}$ Significantly different from group Con. Group Con, $n=10$; group PFo, $n=3 ;$ group Amyg, $n=4$.

effect of session $\left(F_{(4,60)}=2.876 ; p=0.030\right)$ but no session-by-group interaction $\left(F_{(4,60)}=1.256 ; p=0.283\right)$. An identical pattern of results was found for the frequency of defensive behaviors (mean cumulative frequency of defensive behavior: group Con, 7.12; group Amyg, 2.15; group PFo, 3.6). Post hoc Bonferroni's tests revealed that only group Amyg displayed significantly less defensive behavior than group Con (duration, $p=0.002$; frequency, $p=0.018$ ). Nevertheless, group PFo, when compared with only concurrently tested controls, was also found to display significantly less defensive behavior in the presence of the snake. A repeated-measures ANOVA on the cumulative duration of behaviors for these monkeys (PFo, $n=4$; Con, $n=6$ ) revealed a significant main effect of group (mean cumulative duration of defensive behavior: group Con, 47.2; group PFo, 26.14; $\left.F_{(1,8)}=8.630 ; p=0.019\right)$.

Approach behavior. On average, both operated groups displayed a greater amount of approach behavior, relative to controls, in the presence of the snake. Relative to monkeys in group Con, which displayed only $9.4 \mathrm{~s}$ of approach behaviors, monkeys in group Amyg engaged in $27.0 \mathrm{~s}$ and those in group PFo in $15.6 \mathrm{~s}$ of approach behavior. A repeated-measures ANOVA on approach behavior across all five sessions resulted in a main effect of group $\left(F_{(2,15)}=7.915 ; p=0.005\right)$ and a within-subjects effect of session $\left(F_{(4,60)}=3.674 ; p=0.010\right)$ but no session-by-group interaction $\left(F_{(4,60)}=1.522 ; p=0.169\right)$. Post hoc Bonferroni's comparisons revealed that only group Amyg spent significantly more time in approach behavior than group Con $(p=0.004)$. Unlike the significant results for duration, there was no difference in the frequency of approach behaviors across groups (mean cumulative frequency of approach behavior: group Con, 1.46; group Amyg, 2.35; group PFo, 1.8). Group PFo, when compared with only concurrently tested controls, was also found to spend significantly more time in approach behavior. A repeated-measures ANOVA on the cumulative duration of behaviors revealed a significant main effect of group (mean cumulative duration of approach behavior: group Con, 7.476; group PFo, 15.58; $F_{(1,8)}=$ $6.882 ; p=0.030)$.

\section{Experiment 2}

Responses to human intruder

Mean cumulative durations of the different categories of behavior exhibited in the different conditions are shown in Fig- ure 4. Consistent with other published reports (Kalin et al., 2001), intact control monkeys showed more defensive behavior, especially freezing, in the NEC condition relative to the Alone condition. In addition, they showed more defensive behavior in the ST relative to the Alone condition, with a slight increase in aggressive behavior as well. A repeated-measures ANOVA on all three conditions yielded significant interactions of group (Con, PFo, Amyg) by condition (Alone, NEC, $\mathrm{ST})$ for mild aggression $\left(F_{(2,28)}=4.501 ; p=0.006\right)$ and for other behaviors $\left(F_{(4,28)}=3.336 ; p=0.024\right)$. Follow-up tests revealed a significant group difference in mild aggression specific to the stare condition $\left(F_{(2,14)}=4.041 ; p=0.041\right)$. Group PFo spent more time on average in mild aggression in this condition than the other two groups (post hoc Bonferroni's tests; PFo vs Amyg, $p=0.054$; PFo vs Con, $p=0.078$ ), whereas group Amyg and group Con did not differ from each other $(p=1.0)$. A significant group difference also emerged for other behaviors $\left(F_{(2,14)}=7.308 ; p=0.007\right)$, and further analysis showed that group PFo spent significantly less time than group Con in this category ( post hoc Bonferroni's test, $p=0.006$ ), but group PFo did not differ from group Amyg $(p=0.104)$. Group Amyg and group Con also did not differ from each other $(p=1.0)$. (Analyses in which group PFo was compared with only its own concurrently tested controls similarly revealed significant group-bycondition interactions for mild aggression and other behaviors.)

\section{Discussion}

The present study revealed that PFo and amygdala damage produced similar effects on monkeys' responses to a potential predator (rubber snake) but slightly different effects when monkeys were confronted with an unfamiliar human (human intruder). Thus, amygdala and PFo damage caused both similar and different effects on emotional behavior.

\section{Responses to a fake snake}

Monkeys with bilateral PFo lesions had significantly shorter latencies to reach over the rubber snake relative to unoperated controls. In addition, an analysis restricted to group PFo and concurrently tested controls revealed a significant attenuation of 
defensive responses and an increase in approach responses in the presence of the fake snake. Monkeys with amygdala lesions exhibited the same pattern of behaviors as monkeys with PFo lesions. Thus, when monkeys face potential predators, the effects of PFo and amygdala damage are similar, both qualitatively and quantitatively. Importantly, in neither group can the deficit be accounted for by changes in visual perceptual abilities, motivation, or appreciation of foodstuffs (Baxter et al., 2000; Izquierdo et al., 2004). The present results thus provide new information on the effects of PFo damage and, in addition, confirm and extend previous findings of blunted defensive behavior in adult macaques after selective amygdala lesions (Meunier et al., 1999; Kalin et al., 2001) to include shorter than normal food-retrieval latencies in the presence of another prepotently aversive stimulus, a spider.

Neuroimaging investigations indicate the involvement of these same brain regions in phobic subjects in response to their phobic stimulus. For example, regional cerebral blood flow (rCBF) increases in the left posterior orbital cortex while subjects view phobic objects, compared with baseline (Rausch et al., 1995; Reiman, 1997). In addition, using a habituation design, rCBF decreases bilaterally in the amygdala (Veltman et al., 2004) but increases in the posterior orbital cortex (Drevets et al., 1995). And although $\mathrm{rCBF}$ in the right amygdala during exposure to phobic stimuli correlates positively with the phobic individual's subjective assessment of fear (Fredrikson and Furmark, 2003), rCBF increases in posterior orbital cortex are inversely related to changes in heart rate and anxiety levels (Drevets et al., 1995). These and other findings (Timms, 1977) suggest that PFo neurons may inhibit activity in other structures, such as the amygdala, which in turn might suppress expression of emotion or prepotent response tendencies.

\section{Responses to a human intruder}

Relative to controls, monkeys in group PFo showed significantly more mild aggression toward an unfamiliar human in the Stare condition and fewer behaviors in the other category in the Alone condition. Monkeys in group Amyg showed no such alterations in behavior. In support of these negative results, Kalin et al. (2001) likewise found no effect of bilateral, selective amygdala damage on responses to a human intruder. In the Stare condition, unlike the NEC condition, the threat is unambiguous because the intruder makes direct eye contact and maintains it for several minutes. Although the group difference in mild aggression is modest, it is consistent with evidence relating PFo damage (but not amygdala damage) to increased aggression (Grafman et al. 1996) and to the clinical phenomena of reactive aggression and acquired sociopathy (Blair, 2001). These findings, together, suggest a role for the PFo in modulating aggression in threatening situations.

The disruption of emotional responses to snakes cannot be ascribed to an inability to produce the responses. Whereas monkeys with damage to either the PFo or the amygdala exhibited a paucity of emotional responses in the presence of the artificial snake, they made many of the same behaviors in response to the human intruder. For example, in the human intruder task, but not the snake task, both groups of operated monkeys displayed freezing behavior, as well as head and eye aversions, in amounts equivalent to the controls.

Our results contrast with previous reports (Butter et al., 1970; Butter and Snyder, 1972) in which monkeys with PFo damage were reported to show a decrease in aggression and an increase in avoidance behavior in response to human observers. These dif- ferent results could be related to differences in the degree of familiarity of human observers or low interrater reliability for behavioral scoring (Butter et al., 1968, 1970). Regardless, the present study is the first to show different effects of PFo and amygdala damage on responses to social stimuli. Whether this represents a fundamental difference in processing of innate versus conditioned stimuli, predator versus social stimuli, or other differences requires additional study.

\section{Role of the PFo in response selection}

As noted at the outset, evidence from ablation and physiological studies is consistent with a role for the PFo in rule-based response selection, inhibitory control, and representation of the affective value of goals (for review, see Holland and Gallagher, 2004). How the role of the PFo in emotional behavior might relate more broadly to response selection or inhibitory control has not been systematically explored. In the present assessments of emotional responses to a fake snake and human intruder, as in many other tests of instrumental responses in which a context guides action, PFo damage disrupted the elicitation of adaptive responses. Because the actions themselves are available and apparently normally executed, the impairment is clearly in either selecting or guiding action rather than in motor control per se.

The idea that PFo damage leads to either perseveration or loss of inhibitory control, although consistent with some findings, cannot explain the constellation of impairments that follow PFo damage. For example, although monkeys with PFo damage show perseveration on an object discrimination reversal task (Jones and Mishkin, 1972), in another study, monkeys with lesions that included the PFo showed no such perseveration. Instead, they distributed their responses randomly across the possible choices (Bussey et al., 2001). Accordingly, perseveration, per se, cannot account for the deficits that follow PFo damage. Similarly, although deficits in reversal learning or extinction have been characterized as deficits in inhibitory control, monkeys with PFo lesions were unimpaired on a difficult test of inhibitory control, the reversed-contingency task (Y. Chudasama, J. D. Kralik, and E. A. Murray, unpublished observations). Consequently, an account in terms of deficient inhibitory control mechanisms fails to capture the deficits that follow PFo damage.

Several lines of evidence support the idea that the PFo is important for facultative choices based on the value of expected goals. A recurring theme is that the PFo is critical in conditions in which there are multiple competing responses that must be evaluated and selected among. For example, recent work has shown that PFo damage disrupts monkeys' abilities to choose between two objects on the basis of the underlying food value (Izquierdo et al., 2004). In addition, rats with lesions of the orbital frontal cortex are impaired relative to controls in making choices between two actions, one leading to small, immediate rewards and the other to large, delayed rewards (Kheramin et al., 2002; Winstanley et al., 2004). Furthermore, human patients with damage that includes the PFo exhibit impairments in laboratory-based gambling tasks in which they must choose among actions that differ in terms of size and probabilities of punishments and rewards (Bechara et al., 1999, 2000; Rogers et al., 1999). Consistent with these findings, physiological studies have demonstrated that neurons in the PFo code the value of predicted rewards (Tremblay and Schultz, 2000; Wallis and Miller, 2003; Roesch and Olson, 2004), taking into account not only magnitude of the reward (Wallis and Miller, 2003) but also potential punishments (Roesch and Olson, 2004) in assigning value. Thus, PFo neurons appear to integrate different types of sensory information to code the value of expected goals. 
The responses of monkeys with PFo lesions to artificial snakes may inform the possible role of the PFo in integrating sensory inputs. First, on each trial, the positive and negative signals, namely food and the snake, are in full view of the monkey. There is no working memory requirement. Thus, the present results, like the findings from several ablation (Bussey et al., 2001; Rushworth et al., 1997) and physiological (Lebedev et al., 2004; Passingham and Sakai, 2004) studies, argue against the idea that the primary function of the prefrontal cortex is the mediation of working memory. Instead, the deficit after PFo lesions must be in another domain. Second, because both snakes and food are unconditioned stimuli, there is no requirement to associate environmental cues with positive and negative reinforcers. Indeed, observations of these same monkeys in other settings indicate that monkeys with PFo lesions, like controls, display intact food preferences for familiar foods. Thus, the critical role of the PFo lies not in assigning value to food rewards but in another domain. By elimination, then, the best characterization of the role of the PFo in the snake test is that of integrating competing signals to generate appropriate responses. That is, the PFo may be critical for gauging the threat signaled by the snake against the benefit of the food. On this view, the primary role of the PFo would be in providing a common currency for the valuation of goals leading to response selection, rather than in resolving competing response options per se.

\section{References}

Aggleton JP, Passingham RE (1981) Syndrome produced by lesions of the amygdala in monkeys (Macaca mulatta). J Comp Physiol Psychol 95:961-977.

Baxter MG, Parker A, Lindner CCC, Izquierdo AD, Murray EA (2000) Control of response selection by reinforcer value requires interaction of amygdala and orbital prefrontal cortex. J Neurosci 20:4311-4319.

Bechara A, Damasio H, Damasio AR, Lee GP (1999) Different contributions of the human amygdala and ventromedial prefrontal cortex to decision making. J Neurosci 19:5473-5481.

Bechara A, Tranel D, Damasio H (2000) Characterization of the decisionmaking deficit of patients with ventromedial prefrontal cortex lesions. Brain 123:2189-2202.

Blair RJ (2001) Neurocognitive models of aggression, the antisocial personality disorders, and psychopathy. J Neurol Neurosurg Psychiatry 71:727-731.

Bussey TJ, Wise SP, Murray EA (2001) The role of ventral and orbital prefrontal cortex in conditional visuomotor learning and strategy use in rhesus monkeys (Macaca mulatta). Behav Neurosci 115: 971-982.

Butter CM, Snyder DR (1972) Alterations in aversive and aggressive behaviors following orbital frontal lesions in rhesus monkeys. Acta Neurobiol Exp (Wars) 32:525-565.

Butter CM, Mishkin M, Mirsky AF (1968) Emotional responses toward humans in monkeys with selective frontal lesions. Physiol Behav 3:213-215.

Butter CM, Snyder DR, McDonald JA (1970) Effects of orbital frontal lesions on aversive and aggressive behaviors in rhesus monkeys. J Comp Physiol Psychol 72:132-144.

Drevets WC, Simpson JR, Raichle ME (1995) Regional blood flow changes in response to phobic anxiety and habituation. J Cereb Blood Flow Metab 15:S856.

Fredrikson M, Furmark T (2003) Amygdaloid regional cerebral blood flow and subjective fear during symptom provocation in anxiety disorders. Ann NY Acad Sci 985:341-347.

Gallagher M, McMahan RW, Schoenbaum G (1999) Orbitofrontal cortex and representations of incentive value in associative learning. J Neurosci 19:6610-6614.
Grafman J, Schwab K, Warden D, Pridgen A, Brown HR, Salazar AM (1996) Frontal lobe injuries, violence, and aggression: a report of the Vietnam Head Injury Study. Neurology 46:1231-1238.

Hatfield T, Han J-S, Conley M, Gallagher M, Holland P (1996) Neurotoxic lesions of basolateral, but not central, amygdala interfere with pavlovian second-order conditioning and reinforcer devaluation effects. J Neurosci 16:5256-5265.

Holland PC, Gallagher M (2004) Amygdala-frontal interactions and reward expectancy. Curr Opin Neurobiol 14:148-155.

Izquierdo A, Murray EA (2004) Combined unilateral lesions of the amygdala and orbital prefrontal cortex impair affective processing in rhesus monkeys. J Neurophysiol 91:2023-2039.

Izquierdo A, Suda RK, Murray EA (2004) Bilateral orbital prefrontal cortex lesions in rhesus monkeys disrupt choices guided by both reward value and reward contingency. J Neurosci 24:7540-7548.

Jones B, Mishkin M (1972) Limbic lesions and the problem of stimulusreinforcement associations. Exp Neurol 36:362-377.

Kalin NH, Shelton SE (1989) Defensive behaviors in infant rhesus monkeys: environmental cues and neurochemical regulation. Science 243: $1718-1721$

Kalin NH, Shelton SE, Davidson RJ, Kelley AE (2001) The primate amygdala mediates acute fear but not the behavioral and physiological components of anxious temperament. J Neurosci 21:2067-2074.

Kheramin S, Body S, Mobini S, Ho M-Y, Velázquez-Martinez DN, Bradshaw CM, Szabadi E, Deakin JFW, Anderson IM (2002) Effects of quinolinic acid-induced lesions of the orbital prefrontal cortex on inter-temporal choice: a quantitative analysis. Psychopharmacology 165:9-17.

Lebedev MA, Messinger A, Kralik JD, Wise SP (2004) Representation of attended versus remembered locations in prefrontal cortex. PLoS Biol 2:1919-1935.

Malkova L, Gaffan D, Murray EA (1997) Excitotoxic lesions of the amygdala fail to produce impairments in visual learning for auditory secondary reinforcement but interfere with reinforcer devaluation effects in rhesus monkeys. J Neurosci 17:6011-6020.

Malkova L, Lex CK, Mishkin M, Saunders RC (2001) MRI-based evaluation of locus and extent of neurotoxic lesions in monkeys. Hippocampus 11:361-370.

Meunier M, Bachevalier J, Murray EA, Malkova L, Mishkin M (1999) Effects of aspiration versus neurotoxic lesions of the amygdala on emotional responses in monkeys. Eur J Neurosci 11:4403-4418.

Mineka S (1987) A primate model of phobic fears. In: Theoretical foundations of behavior therapy (Eysenck HJ, Martin I, eds), pp 81-111. New York: Plenum.

Mineka S, Keir R, Price V (1980) Fear of snakes in wild- and laboratoryreared rhesus monkeys (Macaca mulatta). Anim Learn Behav 8:653-663.

Nelson EE, Shelton SE, Kalin NH (2003) Individual differences in the responses of naïve rhesus monkeys to snakes. Emotion 3:3-11.

Nemanic S, Alvarado MC, Price RE, Jackson EF, Bachevalier J (2002) Assessment of locus and extent of neurotoxic lesions in monkeys using neuroimaging techniques: a replication. J Neurosci Methods 121:199-209.

Passingham RE, Sakai K (2004) The prefrontal cortex and working memory: physiology and brain imaging. Curr Opin Neurobiol 14:163-168.

Preuss TM, Goldman-Rakic PS (1991) Myelo- and cytoarchitecture of the granular frontal cortex and surrounding regions in the strepsirhine primate Galago and the anthropoid primate Macaca. J Comp Neurol 310:429-474.

Rausch SL, Savage CR, Alpert NM, Miguel EC, Baer L, Breiter HC, Fischman AJ, Manzo PA, Moretti C, Jenike MA (1995) A positron emission tomographic study of simple phobia symptom provocation. Arch Gen Psychiatry $52: 20-28$.

Reiman EM (1997) The application of positron emission tomography to the study of normal and pathological emotions. J Clin Psychiatry 58 [Suppl $16]: 4-12$.

Roesch MR, Olson CR (2004) Neuronal activity related to reward value and motivation in primate frontal cortex. Science 304:307-310.

Rogers RD, Everitt BJ, Baldacchino A, Blackshaw AJ, Swainson R, Wynne K, Baker NB, Hunter J, Carthy T, Booker E, London M, Deakin JFW, Sahakian BJ, Robbins TW (1999) Dissociable deficits in the decision-making cognition of chronic amphetamine abusers, opiate abusers, patients with 
focal damage to prefrontal cortex, and tryptophan-depleted normal volunteers: evidence for monoaminergic mechanisms. Neuropsychopharmacology 20:322-339.

Rushworth MFS, Nixon PD, Eacott MJ, Passingham RE (1997) Ventral prefrontal cortex is not essential for working memory. J Neurosci 17:4828-4838

Schoenbaum G, Setlow B, Saddoris MP, Gallagher M (2003) Encoding predicted outcome and acquired value in orbitofrontal cortex during cue sampling depends upon input from basolateral amygdala. Neuron 39:855-867.

Timms RJ (1977) Cortical inhibition and facilitation of the defense reaction. J Physiol (Lond) 266:98-99.

Tremblay L, Schultz W (2000) Modifications of reward expectation-related neuronal activity during learning in primate orbitofrontal cortex. J Neurophysiol 83:1877-1885.

Veltman DJ, Tuinebreijer WE, Winkelman D, Lammertsma AA, Witter MP, Dolan RJ, Emmelkamp PMG (2004) Neurophysiological correlates of habituation during exposure in spider phobia. Psychiatry Res 132:149-158.

Walker AE (1940) A cytoarchitectural study of the prefrontal area of the macaque monkey. J Comp Neurol 73:59-86.

Wallis JD, Miller EK (2003) Neuronal activity in primate dorsolateral and orbital prefrontal cortex during performance of a reward preference task. Eur J Neurosci 18:2069-2081.

Winstanley CA, Theobald DE, Cardinal RN, Robbins TW (2004) Contrasting roles of basolateral amygdala and orbitofrontal cortex in impulsive choice. J Neurosci 19:4718-4722. 